\title{
Polydopamine Particles Effect on Melanoma Cells Proliferation and Melanin Secretion
}

\author{
Sandy Eap $^{1 *}$, Alice Ferrand ${ }^{1 *}$, Valérie Machi ${ }^{1,2}$, Vincent Ball ${ }^{2}$, Olivier Huck ${ }^{1,2}$, \\ Nadia Benkirane-Jessel ${ }^{1,2 \#}$ \\ ${ }^{1}$ INSERM, Institut National de la Santé et de la Recherche Médicale, \\ "Osteoarticular and Dental Regenerative Nanomedicine" Team, Strasbourg, France \\ ${ }^{2}$ Université de Strasbourg, Faculté de Chirurgie Dentaire, Strasbourg, France \\ Email: "nadia.jessel@inserm.fr
}

Received March 27, 2013; revised April 27, 2013; accepted May 27, 2013

Copyright (C) 2013 Sandy Eap et al. This is an open access article distributed under the Creative Commons Attribution License, which permits unrestricted use, distribution, and reproduction in any medium, provided the original work is properly cited.

\begin{abstract}
Melanin is a biopolymer implicated in the protection of cellular membranes and DNA produced by melanocytes. This pigment has a dual role and should be considered as a photo-protector and as a photosensitizer due to its interaction with UV. The design of multifunctional and biologically responsive coatings is of major interest in modern biomaterials science. The aim of this study is not only to characterize the deposition of multilayered polyelectrolytes films made from polydopamine particles and polyamines like poly-(L-lysine hydrobromide) (PLL), but also to evaluate melanoma cells activity in terms of proliferation and their capacity to stimulate melanin secretion. One could expect that the presence of a melanin like material in the film may have a positive or a negative feedback on the melanin biosynthesis and consequently on melanoma development. Some comparisons are also done with pure polydopamine grains in suspension in the cell culture medium, to investigate if the immobilization of the polydopamine grains has an influence on their bioactivity.
\end{abstract}

Keywords: Polydopamine Particles; Polyelectrolyte Multilayers Films; Melanin; Melanoma Cells

\section{Introduction}

The design of multifunctional and biologically responseve coatings is of major interest in modern biomaterials science [1]. Such coatings should be biodegradable and porous [2] and should display adequate mechanical properties since adherent cells are sensitive to the stiffness of their adhesion substrate [3]. Additionally, coatings useful for bio-applications should contain molecules able to be released in a progressive manner or some growth factors able to interact with the cells in contact with them [4]. Three of the most popular surface functionalization methods consist in the deposition of hydrogels [5], of polyelectrolyte multilayer (PEM) films [6] or thin polydopamine films [7-10] which are chemically and structurally close to natural melanin [11], the natural pigment of skin.

Melanocytes have the ability to produce melanin. Melanin is a biopolymer implicated in the protection of cellular membranes and DNA. This pigment has a dual

\footnotetext{
${ }^{*}$ These authors contributed equally to this work.

${ }^{\text {\#} C o r r e s p o n d i n g ~ a u t h o r . ~}$
}

role and should be considered as a photo-protector and as a photosensitizer due to its interaction with UV [12] but has also been linked to Parkinson's disease characterized by loss of pigmented dopamine neurons in substantia nigra [13].

Films made through spin coating of melanin particles are adequate for adhesions of neurons [14]. However polydopamine films, even if they are advantageous for secondary biofunctionalisation with molecules containing thiol or amino functionalities $[15,16]$, are highly impermeable to ions [17] and molecules of higher molecular mass. Hence it would be advantageous to combine the intrinsic properties of polydopamine with those of hydrogels or PEM films. It has already been demonstrated that polydopamine particles produced through oxidation of dopamine in the presence of dioxygen as an oxidant can be deposited in alternation with a quaternary polyamine, namely poly(dimethyldiallyl ammonium chloride) (PDADMAC) [18]. Those films are permeable to small ions and they could be an ideal platform for bio-applications. The aim of this study is not only to characterize the deposition of PEM film made from polydopamine parti- 
cles and polyamines like poly-(L-lysine hydrobromide) (PLL), but also to evaluate melanoma cells activity in terms of proliferation and their capacity to stimulate melanin secretion. One could expect that the presence of a melanin like material in the film may have a positive or a negative feedback on the melanin biosynthesis and consequently on melanoma development. Some comparisons were also done with pure polydopamine grains in suspension in the cell culture medium, to investigate if the immobilization of the polydopamine grains has an influence on their bioactivity. The biological activity of the (PLL-dopamine) $)_{n}$ PEM films was also compared with that of films not containing polydopamine but a negatively charged counterpart, poly(-L-glutamic) acid.

In addition to the interaction of (PLL-polydopamine) films with melanoma, those films could find interesting use as water resistant adhesives because lysine and L-DOPA are the two major amino acids of the Mytilus Edulis Foot Proteins, Mefp 3 and Mefp 5 which are abundant at the extremity of the mussel byssus plaque [19].

\section{Materials and Methods}

\subsection{Materials}

Poly(L-lysine)-hydrobromide (PLL, $30 \mathrm{kDa}$ ) and polyL-glutamic acid (PGA, $54 \mathrm{kDa}$ ) were purchased from Sigma. Dopamine in the hydrochloride form was also from Sigma (ref H-8502) and was used without further purification. Dopamine was solubilized at $2 \mathrm{~g} \cdot \mathrm{L}^{-1}$ in 50 $\mathrm{mM}$ Tris buffer (Prolabo, France) at $\mathrm{pH} 8.5$ and this solution was vigorously shaken $(300 \mathrm{rpm}$ with a magnetic stirrer) in an open air reactor during $24 \mathrm{~h}$. This allowed to produce a suspension of polydopamine grains. These grains have been characterized by means of Transmission Electron Microscopy in a previous paper [20]. The obtained grains were highly anisotropic in their shape, displaying a disk like morphology with a lateral dimension of about $100-300 \mathrm{~nm}$ and a thickness of only a few $\mathrm{nm}$. To increase its colloidal stability, the suspension was titrated to $\mathrm{pH} 12$ with a concentrated $\mathrm{NaOH}$ solution. Before the beginning of the deposition of each PEM film, the polydopamine suspension was diluted to a final concentration of $0.2 \mathrm{~g} \cdot \mathrm{L}^{-1}$ or too lower concentrations with $10 \mathrm{mM}$ Tris buffer at $\mathrm{pH} 7.5$ and complemented with 150 $\mathrm{mM} \mathrm{NaCl}$. No visible sedimentation of melanin grains occurred in these conditions. Unfortunately, these suspensions were too diluted in polydopamine grains to allow for a characterization by dynamic light scattering.

\subsection{Polyelectrolyte Multilayer Film Preparation}

For all in vitro experiments, PEM films were prepared on glass coverslips (CML, France) cleaned with $10-2 \mathrm{M}$ sodium dodecyl sulfate and subsequently with $0.12 \mathrm{~N}$
$\mathrm{HCl}$ for $15 \mathrm{~min}$ at $100^{\circ} \mathrm{C}$, and then extensively rinsed with deionized water. Glass coverslips were deposited in 24-well plates (Nunc, Danemark). Films having the nominal dipping sequence of (PLL-polydopamine $)_{5}$ or $(\text { PLL-PGA })_{5}$ were prepared by alternated immersion of the pre-treated coverslips during $5 \mathrm{~min}$ in polyelectrolyte solutions $(300 \mu \mathrm{L})$ at the concentration of $50 \mu \mathrm{g} \cdot \mathrm{mL}^{-1}$ for PLL or PGA, and $200 \mu \mathrm{g} \cdot \mathrm{mL}^{-1}$ for polydopamine in the presence of $10 \mathrm{mM}$ Tris buffer at $\mathrm{pH} 7.5$, complemented with $150 \mathrm{mM}$ of $\mathrm{NaCl}$. After each deposition step, the coverslips were rinsed one times for 5 min with $0.15 \mathrm{M}$ $\mathrm{NaCl} 10 \mathrm{mM}$ Tris at $\mathrm{pH}$ 7.5. All the films were sterilized for 30 min using UV light ( $254 \mathrm{~nm}$ ). The adsorption duration of 5 min per dipping step was shown to be sufficient to reach a steady state surface coverage by means of Crystal Microbalance with Dissipation monitoring (QCM-D).

\subsection{QCM-D}

The deposition of the PEM films was monitored in situ with a quartz crystal microbalance using an axial flow chamber QAFC 302 (QCM-D, D300, Q-Sense, Götenborg, Sweden). QCM works by measuring the resonance frequency shift $(\Delta \mathrm{f})$ of a quartz crystal induced by polyelectrolyte or protein adsorption on to the crystal in comparison to the crystal in contact with buffer. Changes in the resonance frequencies were measured at the third $(v=$ $3)$, the fifth $(v=5)$ and the seventh $(v=7)$ overtones of the quartz crystal, corresponding to frequencies close to $15 \mathrm{MHz}, 25$ and $35 \mathrm{MHz}$ respectively. As a first approximation, a shift in $\Delta \mathrm{f} / v$ can be related to a variation of the mass adsorbed to the crystal. This mass is made of the polyelectrolytes and the polydopamine, the ions eventually bound to the film and the water of hydration that is moved during the shearing motion of the piezolelectric quartz crystal. The Sauerbrey relation [20]:

$$
\Gamma=-\mathrm{C} . \Delta \mathrm{f} / v
$$

relates the reduced frequency changes, $\Delta \mathrm{f} / v$ to the total mass bound to the crystal per unit area, $\Gamma$. $\mathrm{C}$ is a proportionality constant characteristic of the crystal used (in our case: $\mathrm{C}=17.7 \mathrm{ng} \cdot \mathrm{cm}^{-2} \mathrm{~Hz}-1$ as provided by the furnisher). Note that the Sauerbrey equation can only be used for films strongly adhering to the substrate. A good qualitative criterion for the validity of the use of Equation (1) is the superposition of the $\Delta \mathrm{f} / v$ signals for all the considered overtones. The details of the methodology applied in the present work were described elsewhere [21].

The film thickness was calculated from the surface concentration calculated in Equation (1) and from the density of the multilayer film, $\rho$, according to:

$$
\mathrm{d}=\Gamma / \rho
$$

we assumed a density of $1.2 \mathrm{~g} \cdot \mathrm{cm}^{-3}$ for the hydrated mu- 
ltilayer film.

\subsection{Atomic Force Microscopy}

The AFM topographical images were acquired using a Pico-SPM device (Molecular Imaging) at a constant resolution of $512 \times 512$ pixels over an area of $10 \mu \mathrm{m} \times$ $10 \mu \mathrm{m}$ and with a frequency of $1 \mathrm{~Hz}$. The film thickness was evaluated after needle scratching of the (PLL-polydopamine $)_{5}$ films and imaging the surface by scanning the cantilever in the direction perpendicular to the needle scratch.

\subsection{Cell Culture}

Murine melanoma cells (B16-F1) were maintained at $37^{\circ} \mathrm{C}$ in a humidified air- $\mathrm{CO}_{2}(5 \%)$ atmosphere in Dulbecco's modified eagle medium (D-MEM) with $4.5 \mathrm{~g} \cdot \mathrm{L}^{-1}$ D-glucose, L-Glutamine, and Hepes (Invitrogen) supplemented with $10 \%$ heat-inactivated fetal calf serum (FCS, Hyclone), $1 \mathrm{mM}$ Sodium pyruvate (Invitrogen), $100 \mathrm{Um} \cdot \mathrm{L}^{-1}$ of penicillin and $100 \mu \mathrm{g} \cdot \mathrm{mL}^{-1}$ streptomycin (Hyclone). Cells were maintained at $37^{\circ} \mathrm{C}$ in $5 \% \mathrm{CO}_{2}$ humidified atmosphere. Cells were treated with polydopamine particles in solution at different concentrations: 1 , $5,10,25,50,100$ or $200 \mu \mathrm{g} \cdot \mathrm{mL}^{-1}$ and their proliferation and melanin secretion was compared to that of cells adhering to (PLL-PGA) $)_{5}$ and to (PLL-polydopamine) $)_{5}$ polyelectrolyte multilayer films.

\subsection{Cellular Proliferation}

AlamarBlue $^{\circledR}$ (Serotec) was used to assess cellular proli- feration. The AlamarBlue ${ }^{\circledR}$ test is a nontoxic, water-soluble, colorimetric redox indicator that changes color in response to cell metabolism. In this study, 10,000 cells/well were seeded in 24 well plates in cell culture medium and incubated at $37^{\circ} \mathrm{C}$ in a humidified air- $\mathrm{CO}_{2}$ (5\%) on the top of PEM-coated $14 \mathrm{~mm}$ diameter coverslips. The coverslips coated with either the (PLL-PGA) or the (PLL-polydopamine) $)_{5}$ films placed in 24-well plates. After 3 and 7 days of culture, the cells were incubated in $10 \%$ AlamarBlue ${ }^{\circledR} / \mathrm{DMEM}$ solution in a humidified atmosphere at $37^{\circ} \mathrm{C}$ and $5 \% \mathrm{CO}_{2}$. After $4 \mathrm{~h}, 200$ $\mu \mathrm{L}$ of incubation media was transferred to 96 -well plates and measured at 570 and $630 \mathrm{~nm}$ in order to determine the percentage of AlamarBlue ${ }^{\circledR}$ reduction. After the lecture, the $10 \%$ AlamarBlue ${ }^{\circledR} / \mathrm{D}-\mathrm{MEM}$ solution was removed and replaced with classical medium until the next measurement.

\subsection{Melanin Assay}

The melanin cells content was quantified after 3 or 7 days of incubation of cells with different PEM film architectures or after contact with polydopamine in solu- tion. $10^{4}$ cells/well were seeded in 24 well plates in cell culture medium and incubated at $37^{\circ} \mathrm{C}$ in a humidified air- $\mathrm{CO}_{2}(5 \%)$. The melanin supernatant was quantified after 3 and 7 days of incubation of cells. The absorbance of the supernatant was measured at a wavelength of 410 $\mathrm{nm}$ using an Elisa reader. Cell pellets were dissolved in $0.85 \mathrm{M} \mathrm{KOH}, 10 \% \mathrm{DMSO}$ at $80^{\circ} \mathrm{C}$ for $90 \mathrm{~min}$. Optical absorbance was measured at $410 \mathrm{~nm}$ using an Elisa reader. Before each measurement the number of cells was evaluated by numeration in a Neubauer counting chamber. The absorbance was then normalized for each measurement to allow comparison between different culture conditions. The melanin content was evaluated according to a standard curve of melanin concentration. All experiments were performed at least three times, each yielding at least triplicate values. When the cells were put in presence of a solution containing polydopamine, the absorbance measurement corresponds to the absorbance of polydopamine and that of the melanin secreted by the cells. Indeed, polydopamine presents an absorption spectrum without peaks and very similar to that of melanin which is a black pigment [22]. This means that the absorbance red from the cells put in contact with the polydopamine solution corresponds to an overestimation of the amount of melanin secreted by the cells.

\subsection{Immunoflorescence}

B16-F1 cells were rinsed with PBS and fixed with 4\% paraformaldehyde in PBS for 1 hour at $37^{\circ} \mathrm{C}$. The cells were then washed three fold during $10 \mathrm{~min}$ with PBS. After PBS washing, the cells were permeabilized, during one hour at room temperature, with PBS containing 1\% BSA and $0,1 \%$ Triton X100 (PBS-Tx). The cells were then incubated overnight at $4^{\circ} \mathrm{C}$ with primary antibody diluted at 1/200 in PBS-Tx. After overnight incubation at $4^{\circ} \mathrm{C}$, the cells were washed with PBS three fold and incubated with a fluorochrome-conjugated secondary antibody diluted at 1/500 in PBS-Tx for $1 \mathrm{~h}$ at room temperature. AlexaFluor 488 goat anti-mouse (Invitrogen) was used as the secondary antibody. The cells were incubated with AlexaFluor 546-Phalloidin (Invitrogen) diluted at $1 / 100$ for $20 \mathrm{~min}$ at room temperature, and counterstained with DAPI at $200 \mathrm{ng} \cdot \mathrm{mL}^{-1}$ for $5 \mathrm{~min}$ at room temperature. They were then washed with PBS three fold during $10 \mathrm{~min}$. The cells were covered with fluorescent mounting medium (Dako) and analyzed by fluorescence microscopy (Leica DM 4000B).

\subsection{Statistical Analysis}

All values are expressed as mean \pm SEM, and all experiments were repeated at least three times. Statistical analysis was performed using the Mann-Whitney U test. A probability $\mathrm{p}$ value $<0.05$ was considered significant 
to reject the null hypothesis.

\section{Results and Discussion}

\subsection{Deposition of Pem Films}

The deposition of the (PLL-polydopamine) ${ }_{\mathrm{n}}$ PEM films was recorded in situ by means of QCM-D (Figure 1). Within the experimental error, the normalized frequency changes, $\Delta \mathrm{f} / v$, overlap for the different harmonics $(v=3$, $5,7)$ of the shear waves propagating along the piezoelectric crystal (Figure 1(a)). This means that the (PLL-polydopamine $)_{\mathrm{n}}$ coatings behave as rigid films and that the Sauerbrey Equation (1) can be used to calculated the hy-

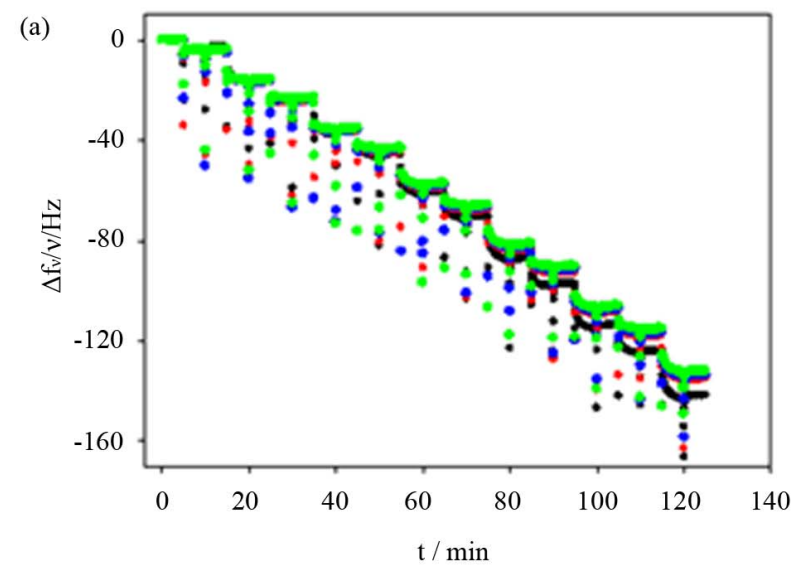

(b)

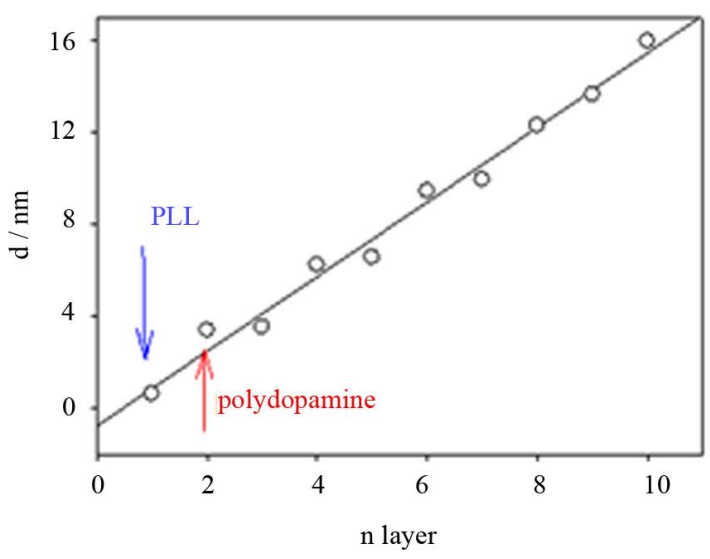

Figure 1. Quartz crystal microbalance measurement to follow in situ the deposition of a (PLL-polydopamine)n film. (a) Reduced frequency shifts at $5(\bullet), 15(\bullet), 25(\bullet)$ and 35 $\mathrm{MHz}(\bigcirc)$ are shown as a function of sequential layer deposition of PLL and polydopamine. (b) The thickness of the coating, calculated according to Equation (2) is plotted as a function of the number of the deposited layer. Odd integers correspond to the deposition of PLL whereas even integers correspond to the deposition of polydopamine particles. The thickness increment corresponding to the first PLL deposition and the first polydopamine deposition are marked with an arrow. The full line corresponds to a linear regression to the experimental data. drated mass of the film as well as the film thickness. The thickness of the (PLL-polydopamine) ${ }_{n}$ coatings increases almost linearly with the number of layer pairs (i.e. one deposition of PLL followed by the deposition of one polydopamine layer) with an average thickness increment of $3.2 \mathrm{~nm}$ per layer pair. However a closer inspection of Figure 1(b) shows that polydopamine contributes more than PLL in the thickness increment of each deposited layer pair.

In addition, the (PLL-polydopamine $)_{n}$ films appear homogeneous and are made from distinct particles about $100 \mathrm{~nm}$ in diameter (Figure 2). This size is in agreement with the size of polydopamine particles produced in solution upon oxygenation of slightly basic dopamine solutions and determined by means of transmission electron microscopy [20]. The thickness of the (PLL-polydoapmine $)_{n}$ films increases proportionally with the number of polycation-polyanion deposition cycles, $n$. This behavior is similar to that found previously for PEM films made from poly(diallyldimethyl ammonium chloride) and polydopamine [18].

The thickness evolution and surface morphology of the $(\mathrm{PLL}-\mathrm{PGA})_{5}$ films, used as a reference for the behavior of melanoma, has been published elsewhere [21].

\subsection{Cellular Proliferation}

The proliferation of melanoma in solution decreases when the concentration of polydopamine in the cell culture medium increases (Figure 3). The polydopamine used in this study is in the form of platelet like particles of about $100-200 \mathrm{~nm}$ in extension and a few $\mathrm{nm}$ in thickness. The effect is more pronounced when the incubation time of the cells with the culture medium containing the polydopamine increases from 3 to 7 days. These data show that increasing dopamine concentration in the cell medium induces a pronounced reduction of their proliferation.
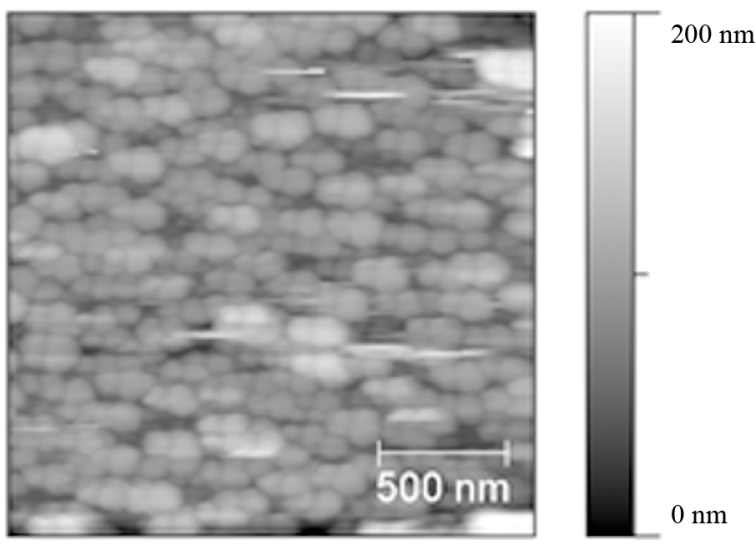

Figure 2. AFM topographical image $(2 \mu \mathrm{m} \times 2 \mu \mathrm{m})$ of a (PLL-polydopamine) $)_{5}$ film deposited on a quartz crystal used for the QCM-D experiments displayed in Figure 1. 


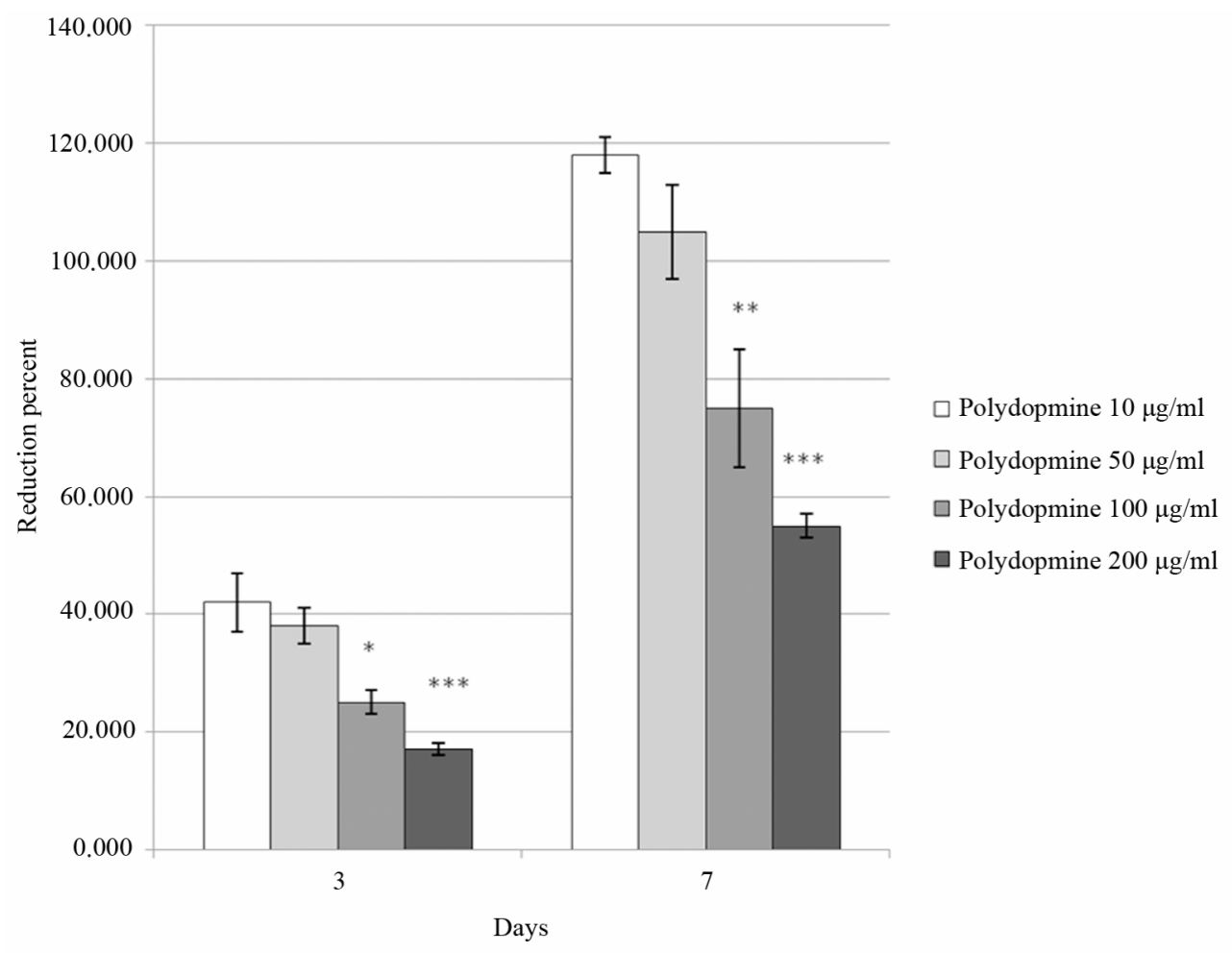

Figure 3. Proliferation of B16F1 murine melanoma cells growing in contact with melanin in solution at various concentrations in the culture medium $\left(10,50,100\right.$ or $\left.200 \mu \mathrm{g} \cdot \mathrm{mL}^{-1}\right)$. Cells were deposited on 24 well plates at a density of $1 \times 10^{4}$ cells/well. Proliferation was measured after 3 days or 7 days of culture with polydopamine in solution and was quantified with the Alamar ${ }^{\circledR}$ Blue test.

On the other hand when polydopamine is present in the PEM films as the last deposited layer, hence with the possibility of the melanine-like material to be in direct contact with the cells, their proliferation is significantly enhanced with respect to the same cells put in contact with (PLL-PGA) ${ }_{5}$ films (Figure 4) which are made from the same polycation, PLL, but differ from the polydopamine containing films with respect to the incorporated polyanion. This result means that contrarily to polydopamine particles present in solution, their immobilization on the surface allows for the melanoma to have a better proliferative behavior. It cannot be excluded that the positive effect of polydopamine is due to some enhancement of the mechanical properties of the (PLL-po${\text { lydopamine })_{5} \text { film with respect to the soft (PLL-PGA) }}_{5}$ film. It is well known that the behavior of cells on surfaces is not only dependent on the composition of that surface but also on its mechanical properties [3]. Indeed, one may expect the (PLL-polydopamine) $)_{\mathrm{n}}$ films to be stiffer than their (PLL-PGA)n counterparts owing to the establishment of covalent bonds between polydopamine and PLL [15]. The quantification of the mechanical properties of the (PLL-polydoamine) $)_{\mathrm{n}}$ films with colloidal probe AFM requires to produce thick coatings in order to avoid a strong influence of the rigid silica substrate. The mechanical properties of these coatings will be investi- gated in a forthcoming investigation.

\subsection{Melanine Secretion}

In the presence of polydopamine in solution, the secretion of melanin by the melanocytic cells is considerably amplified when the polydopamine concentration increases particularly in the supernatant and the more so for shorter incubation times of 3 days (Figure 5).

However, after 3 days of culture, the secretion of melanin by melanoma cells adhering on (PLL-polydopamine $)_{5}$ films is significantly lower than on (PLL-P$\mathrm{GA})_{5}$ films (Figure 6) even if the latter are less favorable than the former ones concerning the cellular proliferation (Figure 4). This result shows that the presence of polydopamine, chemically and structurally similar to melanin, on the PEM films significantly hinders the secretion of melanin by the melanoma cells in presence of the films. This result holds true not only in the cell lysate but also in the supernatant. But after 7 day of culture the difference in melanin secretion by the cells in contact with the (PLL-polydopamine) $)_{5}$ and the (PLL-PGA) films decreases, even if it remains significant. These results could be explained by the limited size of our active nanoreservoir here $(n=5)$. In our group, we have shown that the mechanism by which the cells interact with our 


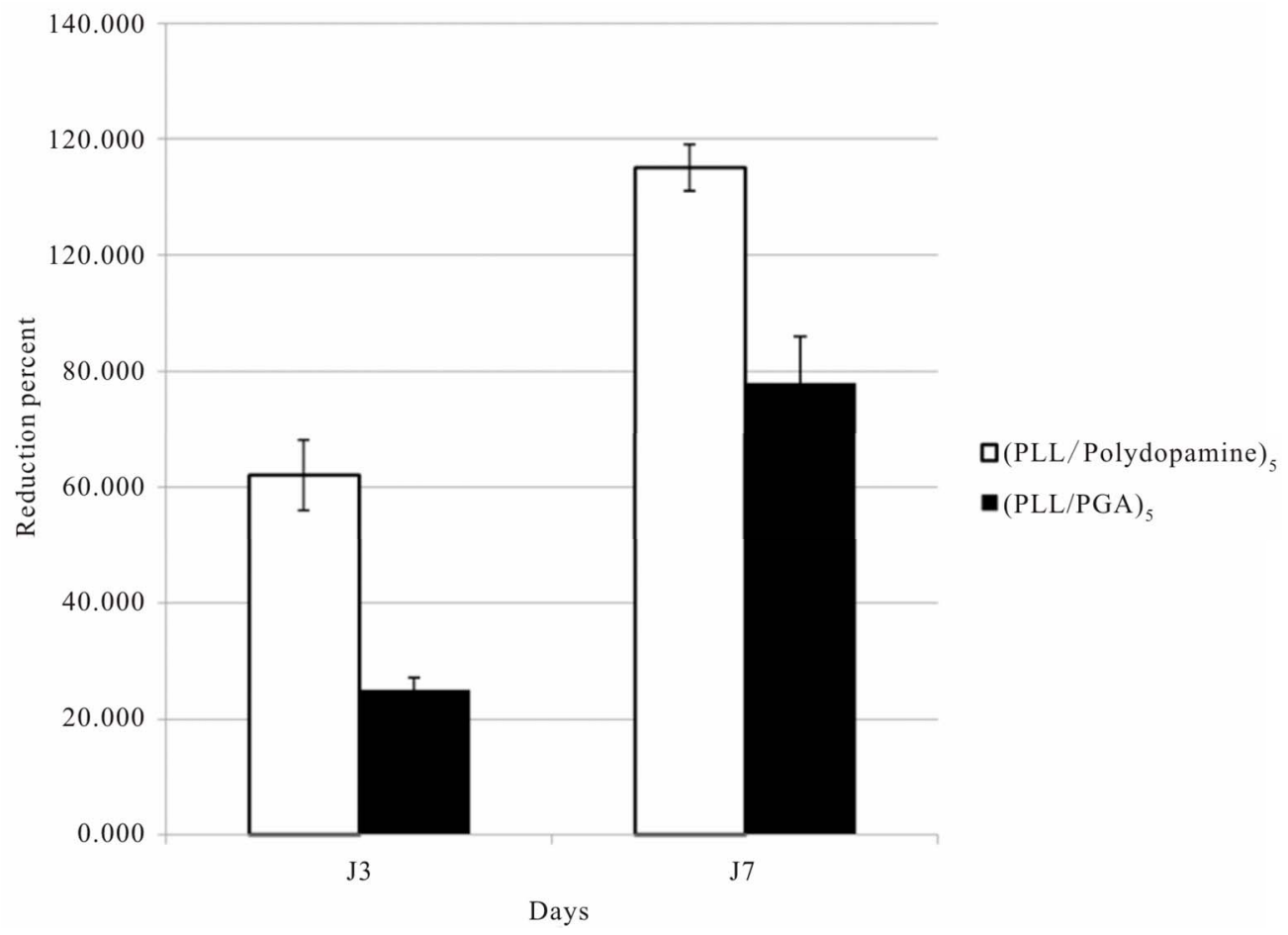

Figure 4. Proliferation of B16F1 murine melanoma cells growing on the surface of (PLL-polydopamine) $)_{5}$ or (PLL-PGA) films. Cells were deposited on 24 well plates at a density of $1 \times 10^{4}$ cells/well. Proliferation was measured after 3 days or 7 days of culture in contact with multilayered films and was quantified by the Alamar ${ }^{\circledR}$ Blue test.

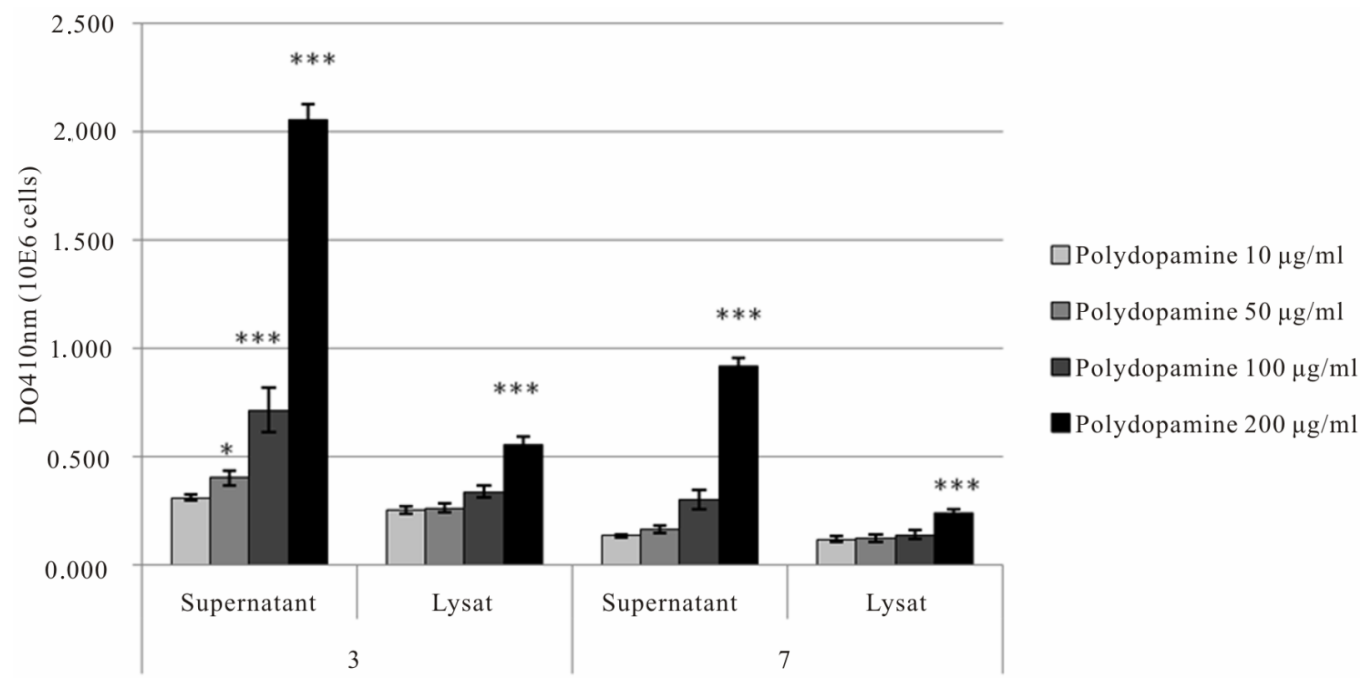

Figure 5. Melanin production by B16F1 Murine melanoma cells growing in contact with polydopamine in solution at different concentration in the culture medium $\left(10,50,100\right.$ or $\left.200 \mu \mathrm{g} \cdot \mathrm{mL}^{-1}\right)$. Cells were deposited on 24 well plates at a density of 1 $\times 10^{4}$ cells/well. The melanin production was measured after 3 or 7 days of culture.

active films is the local degradation by cells. We have also reported that by increasing the size of our reservoir (n), we increase the activity [23-30].

Finally, the morphology and organization of the melanoma cells adhering on both kinds of multilayer films was investigated with staining methods after 3 days (Figure 7) and 7 days (Figure 8) of culture.
Our results indicated clearly here that after 3 days of culture, melanocytics cells adhesion is clearly better onto (PLL-polydopamine $)_{5}$ film than onto (PLL/PGA) $)_{5}$ film. The cells are widespread onto the (PLL-polydopamine) film, their cytoskeleton shown by actin fibers are making as much filopods as possible in comparison to the round and non-spread cells onto the (PLL/PGA) 5 film. After 7 
(a)

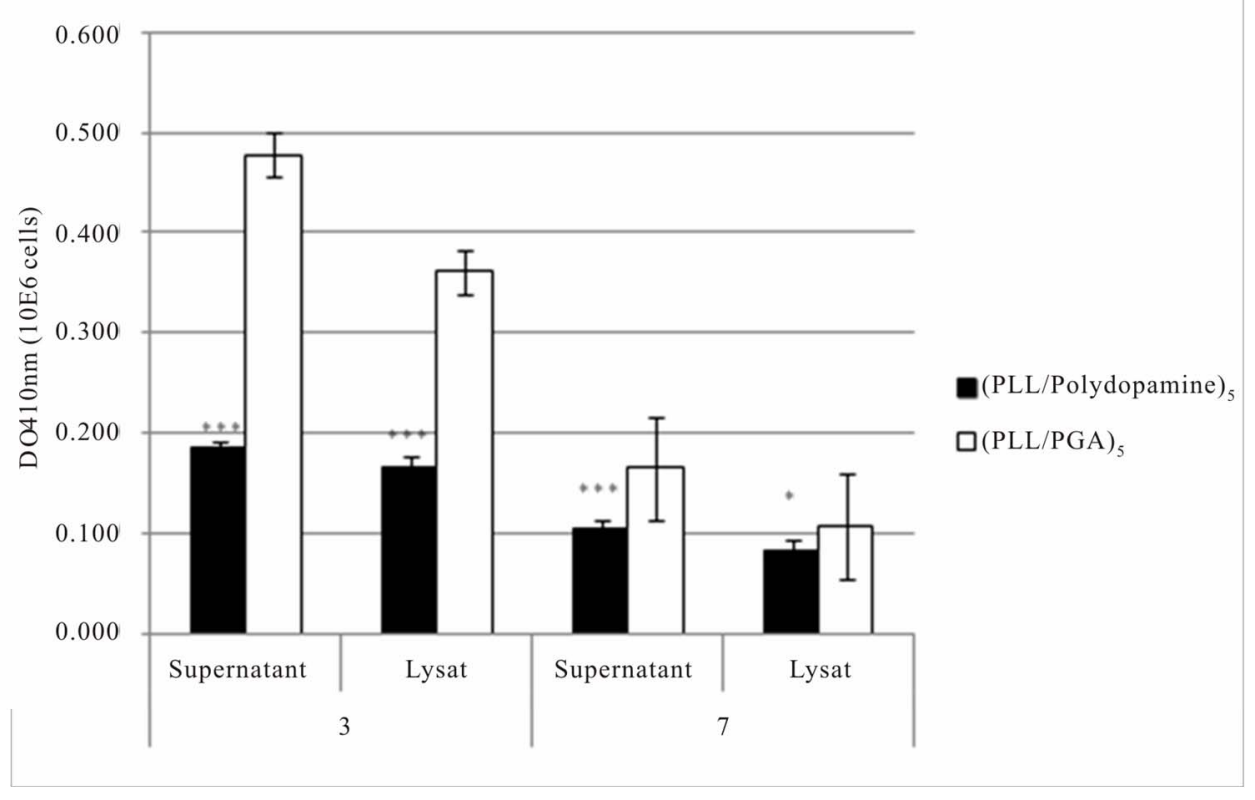

(b)

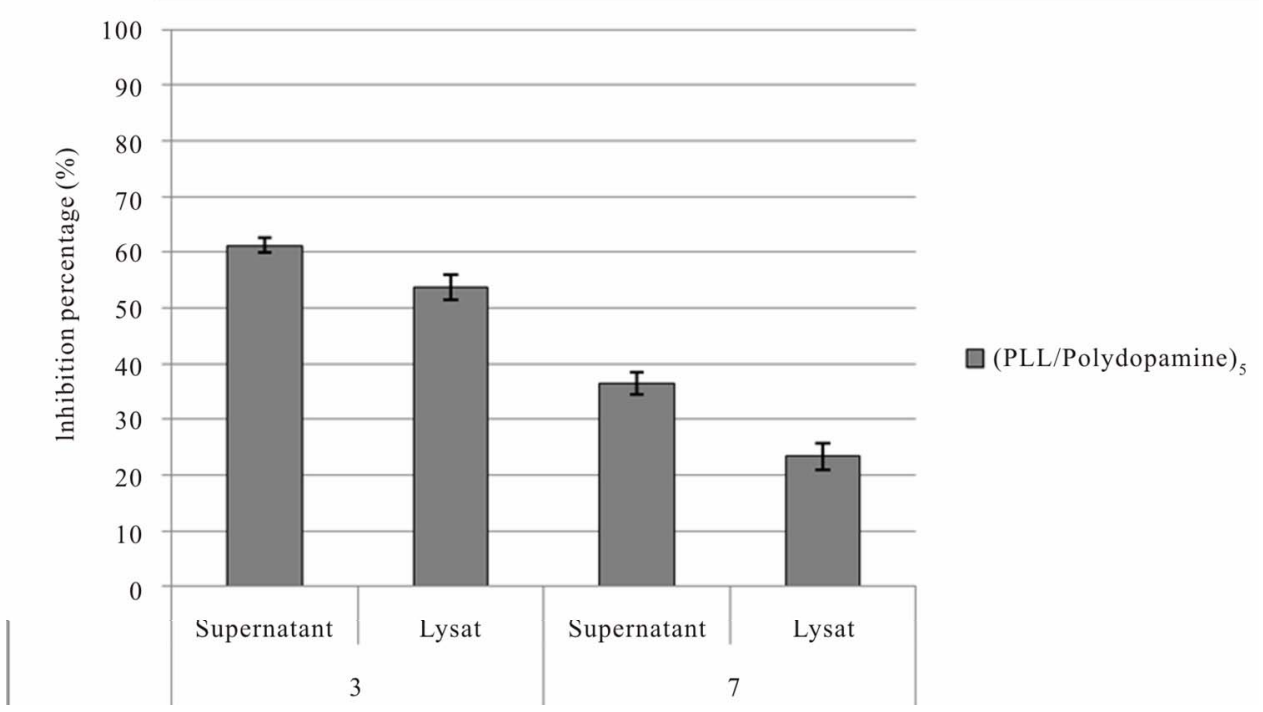

Figure 6. (a) Melanin production by B16F1 murine melanoma cells growing on the surface of the (PLL-polydopamine) $)_{5}$ or (PLL-PGA) 5 films. The cells were deposited on 24 well plates at a density of $1 \times 10^{4}$ cells/well. The melanin production was measured after 3 or 7 days of culture. (b) Percentage of inhibition of melanin productioon by B16F1 murine melanoma cells growing on the surface of the multilayered films (PLL-polydopamine) $)_{5}$ in comparison with (PLL/PGA) 5 films. Cells were deposited on 24 well plates at a density of $1 \times 10^{4}$ cells/well. The melanin production was measured after 3 or 7 days of culture.

days (Figure 8), we have shown that the multiplication and morphology of these cells confirmed this tendency, which clearly means that (PLL-polydopamine) $)_{5}$ film is greatly favorable to our murine melanoma cells adhesion and proliferation as we have observed earlier.

Clearly, our results show that dopamine is more favorable than glutamic acid and promotes good proliferation of these melanocytics cells.

To understand these results, we have also shown that in term of melanin secretion, increasing polydopamine concentration in the medium greatly increases the production of melanin by melanoma cells. However, on the contrary, incorporation of polydopamine in LBL film seems to significantly reduce melanin secretion in comparison to the control film without polydopamine, which means that the inhibition effect of polydopamine on the melanin secretion is not only due to polydopamine sole presence in solution but also to its incorporated and immobilized form into the multilayered polyelectrolyte film. Indeed, incorporated into the LBL film, polydopamine 

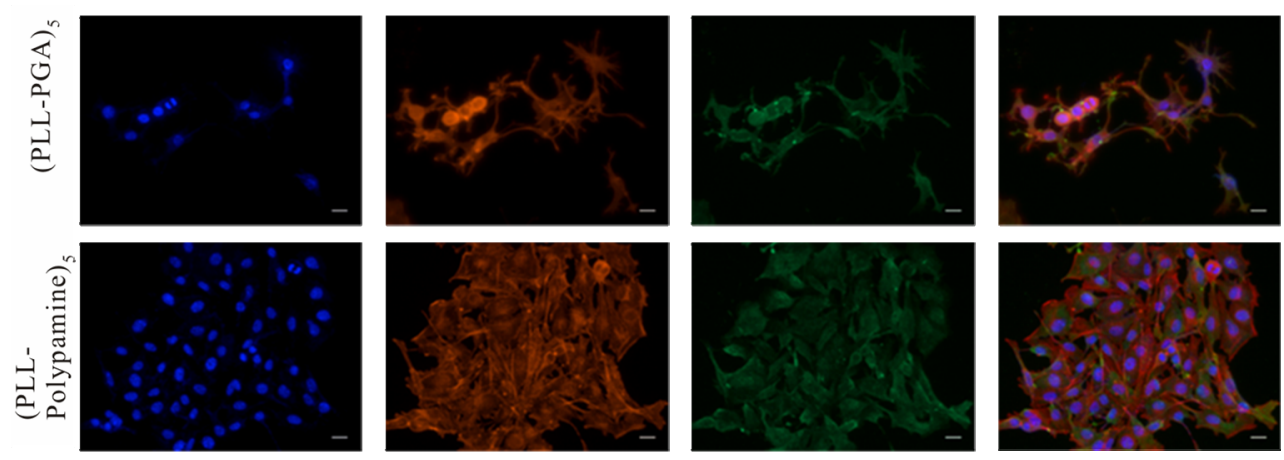

Figure 7. Morphology and organization of B16F1 murine melanoma cells growing on the surface of the multilayered films. Proliferation of B16F1 cells after 3 days of culture on the surface of the (PLL-PGA) 5 or (PLL-polydopamine) 5 films. Morphology and actin organization of cells observed by fluorescent microscopy: Integrin $\alpha v$ (green), Alexa Fluor 546-labeled phalloidin (red) and DAPI (blue), scale bar $=20 \mu \mathrm{m}$.
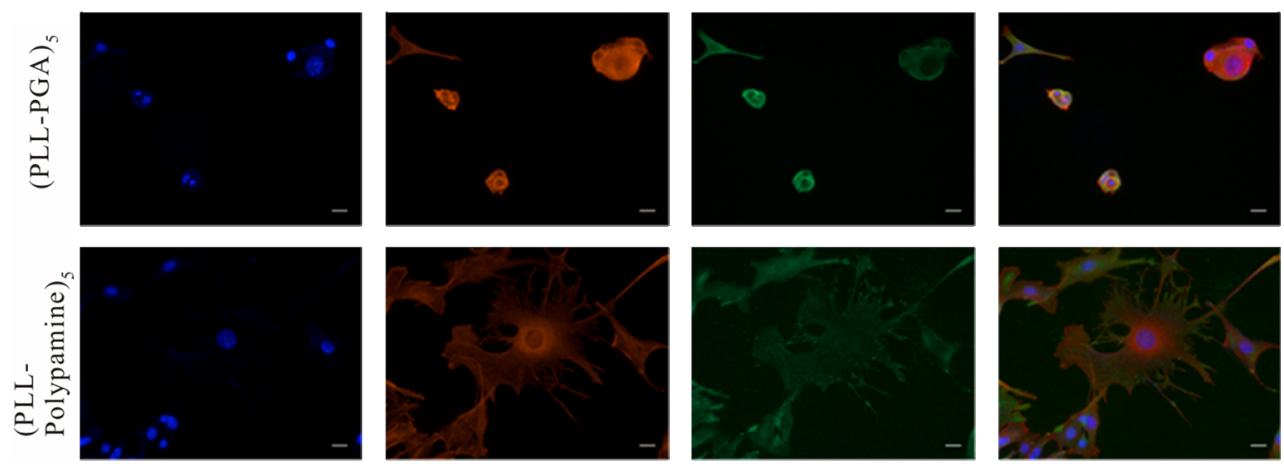

Figure 8. Morphology and organization of B16F1 Murine melanoma cells growing on the surface of the multilayered films. Proliferation of B16F1 cells after 7 days of culture on the surface of (PLL-PGA) 5 or (PLL-Melanin) 5 films. Morphology and actin organization of cells observed by fluorescent microscopy: Integrin $\alpha v$ (green), Alexa Fluor 546-labeled phalloidin (red) and DAPI (blue), scale bar $=20 \mu \mathrm{m}$.

would be directly internalized by melanoma cells throughout the different layers whereas contact between cells and polydopamine in solution is highly limited in comparison.

\section{Conclusion}

As we have shown, even if increasing concentration of polydopamine in solution leads to a decrease in melanoma cells proliferation, it is not the case when polydopamine is incorporated into the LBL film; in contrast, cell proliferation is even higher than the control without polydopamine. However, in terms of melanin secretion, the polydopamine incorporation into the LBL film seems to significantly reduce the production of melanin by melanoma cells in comparison to the effect of polydopamine in solution.

\section{Acknowledgements}

This work was supported by the project Angiomatrix from the "Agence Nationale de la Recherche, ANR" and "Alsace contre le Cancer", C.M. thanks the Faculté de Chirurgie Dentaire of Strasbourg for financial support.
N.J. is indebted to CHU de Nancy, Hôpital Central, "Chirurgie Orthopédique et Traumatologie" (Contrat d'interface INSERM vers l'hôpital).

\section{REFERENCES}

[1] N. A. Peppas and R. Langer, "New Challenges in Biomaterials," Science, Vol. 263, No. 5154, 1994, pp. 17151720. doi: $10.1126 /$ science. 8134835

[2] R. Langer and D. A. Tirrell, "Designing Materials for Biology and Medicine," Nature, Vol. 428, No. 6982, 2004, pp. 487-492. doi:10.1038/nature02388

[3] D. E. Discher, P. Janmey and Y.-L. Wang, "Tissue Cells Feel and Respond to the Stiffness of Their Substrate," Science, Vol. 310, No. 5751, 2005, pp. 1139-1143. doi:10.1126/science.1116995

[4] Y. Hirano and D. J. Mooney, "Peptide and Protein Presenting Materials for Tissue Engineering," Advanced Materials, Vol. 16, No. 1, 2004, pp. 17-25. doi:10.1002/adma.200300383

[5] K. Y. Lee and D. J. Mooney, "Hydrogels for Tissue Engineering," Chemical Review, Vol. 101, No. 7, 2001, pp 1869-1879. doi:10.1021/cr000108x

[6] Z. Tang, Y. Wang, P. Podsiadlo and N. A. Kotov, "Bio- 
medical Applications of Layer-by-Layer Assembly: From Biomimetics to Tissue Engineering," Advanced Materials, Vol. 18, No. 24, 2006, pp. 3203-3224. doi:10.1002/adma.200600113

[7] H. Lee, S. M. Dellatore, W. M. Miller and P. B. Messersmith, "Mussel-Inspired Surface Chemistry for Multifunctional Coatings," Science, Vol. 318, No. 5849, 2007, pp. 426-430. doi:10.1126/science. 1147241

[8] B. Zhu and S. Edmondson, "Polydopamine-Melanin Initiators for Surface-Initiated ATRP," Polymer, Vol. 52, No. 10, 2011, pp. 2141-2149. doi:10.1016/j.polymer.2011.03.027

[9] K. C. Black, J. Yi, J. G. Rivera, D. C. Zelasko-Leon and P. B. Messersmith, "Polydopamine-Enabled Surface Functionalization of Gold Nanorods for Cancer CellTargeted Imaging and Photothermal Therapy," Nanomedicine, Vol. 8, No. 1, 2013, pp. 17-28. doi: $10.2217 / \mathrm{nnm} .12 .82$

[10] M. E. Lynge, R. van der Westen, A. Postma and B. Stadler, "Polydopamine-A Nature-Inspired Polymer Coating for Biomedical Science," Nanoscale, Vol. 3, No. 12, 2011, pp. 4916-4928. doi:10.1039/c1nr10969c

[11] M. d'Ischia, A. Napolitano, A. Pezzella, P. Meredith and T. Sarna, "Chemical and Structural Diversity in Eumelanins: Unexplored Bio-Optoelectronic Materials," Angewandte Chemie International Edition in English, Vol. 48, No. 22, 2009, pp. 3914-3921. doi:10.1002/anie. 200803786

[12] H. Swalwell, J. Latimer, R. M. Haywood and M. A. Birch-Machin, "Investigating the Role of Melanin in UVA/UVB- and Hydrogen Peroxide-Induced Cellular and Mitochondrial ROS Production and Mitochondrial DNA Damage in Human Melanoma Cells," Free Radical Biology and Medicine, Vol. 52, No. 3, 2012, pp. 626-634. doi:10.1016/i.freeradbiomed.2011.11.019

[13] T. Pan, J. Zhu, W.-J. Hwu and J. Jankovic, "The Role of Alpha-Synuclein in Melanin Synthesis in Melanoma and Dopaminergic Neuronal Cells," PLoS ONE, Vol. 7, No. 9, 2012, Artciel ID: e45183. doi:10.1371/journal.pone.0045183

[14] C. J. Bettinger, R. Langer and J. T. Borenstein, "Engineering Substrate Topography at the Micro- and Nanoscale to Control Cell Function," Angewandte ChemieInternational Edition, Vol. 48, No. 30, 2009, pp. 54065415. doi:10.1002/anie.200805179

[15] H. Lee, J. Rho and P. B. Messersmith, "Facile Conjugation of Biomolecules onto Surfaces via Mussel Adhesive Protein Inspired Coatings," Advanced Materials, Vol. 21, No. 4, 2009, pp. 431-434. doi:10.1002/adma.200801222

[16] F. Bernsmann, B. Frisch, C. Ringwald and V. Ball, "Protein Adsorption on Dopamine-Melanin Films: Role of Electrostatic Interactions Inferred from Zeta-Potential Measurements versus Chemisorption," Journal of Colloid Interface Science, Vol. 344, No. 1, 2010, pp. 54-60.

[17] F. Bernsmann, J.-C. Voegel and V. Ball, "Different Synthesis Methods Allow to Tune the Permeability and Permselectivity of Dopamine-Melanin Films to Electrochemical Probes," Electrochimica Acta, Vol. 56, No. 11, 2011, pp. 3914-3919. doi:10.1016/j.electacta.2011.02.028

[18] F. Bernsmann, O. Ersen, J. C. Voegel, E. Jan, N. A. Kotov and V. Ball, "Melanin-Containing Films: Growth from Dopamine Solutions versus Layer-by-Layer Deposition," Chemphyschem, Vol. 11, No. 15, 1002, pp. 3299. 3305.

[19] B. P. Lee, P. B. Messersmith, J. N. Israelachvili and J. H. Waite, "Mussel-Inspired Adhesives and Coatings," Annual Review of Materials Research, Vol. 41, 2011, pp. 99-132.

[20] G. Sauerbrey, "Verwendung von Schwingquarzen zur Wägung dünner Schichten und zur Mikrowägung," Zeitschrift für Physik, Vol. 155, No. 2, 1959, pp. 206-222. doi:10.1007/BF01337937

[21] V. Ball, M. Michel, F. Boulmedais, J. Hemmerle, Y. Haikel, P. Schaaf and J. C. Voegel, "Nucleation Kinetics of Calcium Phosphates on Polyelectrolyte Multilayers Displaying Internal Secondary Structure," Crystal Growth \& Design, Vol. 6, No. 1, 2005, pp. 327-334. doi: $10.1021 / \operatorname{cg} 050044 \mathrm{p}$

[22] P. Meredith, B. J. Powell, J. Riesz, S. P. NighswanderRempel, M. R. Pederson and E. G. Moore, "Towards Structure-Property-Function Relationships for Eumelanin," Soft Matter, Vol. 2, No. 1, 2006, pp. 37-44. doi:10.1039/b511922g

[23] N. Jessel, F. Atalar, P. Lavalle, J. Mutterer, G. Decher, P. Schaaf, J. C. Voegel and J. Ogier, "Bioactive Coatings Based on a Polyelectrolyte Multilayer Architecture Functionalized by Embedded Proteins," Advanced Materials, Vol. 15, No. 9, 2003, pp. 692-695. doi:10.1002/adma.200304634

[24] N. Benkirane-Jessel, P. Lavalle, F. Meyer, F. Audouin, B. Frisch, P. Schaaf, J. Ogier, G. Decher and J. C. Voegel, "Control of Monocyte Morphology on and Response to Model Surfaces for Implants Equipped with Anti-Inflammatory Agents," Advanced Materials, Vol. 16, No. 17, 2004, p. 1507. doi:10.1002/adma.200306613

[25] N. Benkirane-Jessel, P. Schwinte, P. Falvey, R. Darcy, Y. Haikel, P. Schaaf, J. C. Voegel and J. Ogier, "Build-Up of Polypeptide Multilayer Coatings with Anti-Inflammatory Properties Based on the Embedding of PiroxicamCyclodextrin Complexes," Advanced Functional Materials, Vol. 14, No. 2, 2004, pp. 174-182. doi:10.1002/adfm.200304413

[26] N. Jessel, M. Oulad-Abdelghani, F. Meyer, P. Lavalle, Y. Haikel, P. Schaaf and J. C. Voegel, "Multiple and Time-Scheduled in Situ DNA Delivery Mediated by Beta-Cyclodextrin Embedded in a Polyelectrolyte Multilayer," Proceedings of the National Academy of Sciences, Vol. 103, No. 23, 2006, pp. 8618-8621. doi:10.1073/pnas.0508246103

[27] B. S. Kim, S. W. Park and P. T. Hammond, "Hydrogen-Bonding Layer-by-Layer-Assembled Biodegradable Polymeric Micelles as Drug Delivery Vehicles from Surfaces," ACS Nano, Vol. 2, No. 2, 2008, pp. 386-392. doi:10.1021/nn700408z

[28] N. Benkirane-Jessel, P. Lavalle, E. Hubsch, V. Holl, B. Senger, Y. Haikel, J. C. Voegel, J. Ogier and P. Schaaf, "Short-Time Tuning of the Biological Activity of Func- 
tionalized Polyelectrolyte Multilayers," Advanced Functional Materials, Vol. 15, No. 4, 2005, pp. 648-654.

doi:10.1002/adfm.200400129

[29] A. Dierich, E. Le Guen, N. Messaddeq, J.-F. Stoltz, P. Netter, P. Schaaf, J.-C. Voegel and N. Benkirane-Jessel, "Bone Formation Mediated by Synergy-Acting Growth Factors Embedded in a Polyelectrolyte Multilayer Film," Advanced Materials, Vol. 19, No. 5, 2007, pp. 693-697. doi:10.1002/adma.200601271

[30] S. Facca, C. Cortez, C. Mendoza-Palomares, N. Messadeq, A. Dierich, A. P. R. Johnston, D. Mainard, J. C. Voegel, F. Caruso and N. Benkirane-Jessel, "Active Multilayered Capsules for in Vivo Bone Formation," Proceedings of the National Academy of Sciences of the United States of America, Vol. 107, No. 8, 2010, pp. 3406-3411. doi:10.1073/pnas.0908531107 